\title{
ON THE NOTION OF ANALYTICITY
}

\section{F. MOPPERT}

Let $E_{n}$ be a normed $n$-dimensional vector space over a field $\Gamma$. We denote by $a, b, \cdots$ the elements of $E_{n}$, with $\alpha, \beta, \cdots$ the elements of $\Gamma ; a=\alpha_{1} e_{1}+\cdots+\alpha_{n} e_{n} \sim\left\{\alpha_{1}, \cdots, \alpha_{n}\right\}$ where $e_{1}, \cdots, e_{n}$ is a basis of $E_{n}$. The norm in $E_{n}$ we denote by ||$_{n}$.

By $a=L \circ b$ we denote a linear homogeneous transformation on $E_{n}$ to $E_{n}$, i.e. $a \in E_{n}$ is defined for every $b \in E_{n} ; L \circ(\lambda b)=\lambda L \circ b$; $L \circ\left(b_{1}+b_{2}\right)=L \circ b_{1}+L \circ b_{2}$ for every $\lambda, b, b_{1}, b_{2}$. Naturally, given a basis $e_{1}, \cdots, e_{n}$ of $E_{n}, L$ can be represented as a square matrix: $L \sim\left[\lambda_{\mu \nu}\right]$ such that $\alpha_{\mu}=\lambda_{\mu 1} \beta_{1}+\cdots+\lambda_{\mu n} \beta_{n} \quad(\mu=1, \cdots, n)$ for $a=L \circ b, a \sim\left\{\alpha_{1}, \cdots, \alpha\right\}, b=\left\{\beta_{1}, \cdots, \beta_{n}\right\}$.

Let $y=f(x)$ be a function on $E_{n}$ to $E_{n}$, i.e. $y \in E_{n}$ for some $x \in E_{n}$. $f(x)$ is called differentiable (in the sense of Fréchet) at $x=x_{0}$ if for $h \in E_{n},|h|_{n} \rightarrow 0$ there is a $L=L\left(x_{0}\right)$ such that

(1) $f\left(x_{0}+h\right)=f\left(x_{0}\right)+L\left(x_{0}\right) \circ h+r(h) ; r(h) \in E_{n},|r(h)|_{n}=o\left(|h|_{n}\right)$.

If, with respect to the basis $e_{1}, \cdots, e_{n}$ it is $f \sim\left\{\phi_{1}, \cdots, \phi_{n}\right\}$, $x \sim\left\{\xi_{1}, \cdots, \xi_{n}\right\}, L \sim\left[\lambda_{\mu \nu}\right]$ then obviously

$$
\lambda_{\mu \nu}=\partial \phi_{\mu} / \partial \xi_{\nu} \quad\left(x=x_{0}\right) .
$$

We call $\otimes a$ product on $E_{n}$ if to every $a \in E_{n}, b \in E_{n}$ there exists $c=a \otimes b \in E_{n}$ such that $a \otimes b=L_{a} \circ b=L_{b} \circ a$. For some fixed basis $e_{1}, \cdots, e_{n}$ with $a \sim\left\{\alpha_{1}, \cdots, \alpha_{n}\right\}, b \sim\left\{\beta_{1}, \cdots, \beta_{n}\right\}, c \sim\left\{\gamma_{1}, \cdots, \gamma_{n}\right\}$ such a product can always be realized by $n^{3}$ constants $\pi_{\rho \mu \nu}$ such that

$$
\gamma_{\rho}=\sum_{\mu, \nu=1}^{n} \pi_{\rho \mu \nu} \alpha_{\mu} \beta_{\nu} \quad(\rho=1, \cdots, n) .
$$

Naturally, by introducing a product in a vector space this vector space becomes an algebra.

If $f(x)$ is a function on $E_{n}$ to $E_{n}$ we call it analytic with respect to the product $\otimes$ at $x=x_{0}$ if it is there differentiable and if there is an $l=l\left(x_{0}\right) \in E_{n}$ such that

$$
L\left(x_{0}\right) \circ h=l\left(x_{0}\right) \otimes h,
$$
1958.

Received by the editors December 21, 1957 and, in revised form, November 10 , 
where $L\left(x_{0}\right)$ is defined by (1). Without referring to the definition of differentiability this means: there is an $l\left(x_{0}\right)$ such that

$$
\begin{array}{rlrl}
f\left(x_{0}+h\right) & =f\left(x_{0}\right)+l\left(x_{0}\right) \otimes h+r(h), & r(h) \in E_{n}, \\
|r(h)|_{n}=o\left(|h|_{n}\right) & \text { for }|h|_{n} \rightarrow 0 .
\end{array}
$$

It is obvious that this definition of analyticity contains the one for functions of a complex variable.

Let $l\left(x_{0}\right) \sim\left\{\lambda_{1}\left(x_{0}\right), \cdots, \lambda_{n}\left(x_{0}\right)\right\}, h \sim\left\{\eta_{1}, \cdots, \eta_{n}\right\}$ for a basis $e_{1}, \ldots, e_{n}$. Putting $l\left(x_{0}\right) \otimes h=k, k \sim\left\{\kappa_{1}, \cdots, \kappa_{n}\right\}$ we have $\kappa_{\rho}$ $=\sum_{\mu, \nu=1}^{n} \pi_{\rho \mu \nu} \lambda_{\mu}\left(x_{0}\right) \eta_{\nu}(\rho=1, \cdots, n)$. On the other hand, the $\rho$ th component of $L\left(x_{0}\right) \circ h$ is given by

$$
\lambda_{\rho 1} \eta_{1}+\cdots+\lambda_{\rho n} \eta_{n}=\partial \phi_{\rho} / \partial \xi_{1} \cdot \eta_{1}+\cdots+\partial \phi_{\rho} / \partial \xi_{n} \cdot \eta_{n} .
$$

Instead of (4) we have therefore

$$
\partial \phi_{\rho} / \partial \xi_{\nu}=\pi \rho_{1 \nu} \lambda_{1}\left(x_{0}\right)+\cdots+\pi_{\rho n \nu} \lambda_{n}\left(x_{0}\right) \quad(\rho, \nu=1, \cdots, n) .
$$

We put

$$
\Pi_{\nu}=\operatorname{Det} \pi_{\rho \mu \nu}=\left|\begin{array}{lll}
\pi_{11 \nu} & & \\
& \ddots & \\
& & \pi_{n n \nu}
\end{array}\right| \quad(\nu=1, \cdots, n)
$$

and denote by $\Pi_{\nu \rho}$ the determinant we get from $\Pi_{\nu}$ by replacing its $\rho$ th column by $\partial \phi_{1} / \partial \xi_{\nu}, \cdots, \partial \phi_{n} / \partial \xi_{\nu}$. It follows

$$
\Pi_{1 \rho} / \Pi_{1}=\cdots=\Pi_{n \rho} / \Pi_{n}\left(=\lambda_{\rho}\right) \quad(\rho=1, \cdots, n),
$$

provided $\Pi_{\nu} \neq 0(\nu=1, \cdots, n)$. If some of these determinants vanish, $\Pi_{1}=\cdots=\Pi_{k}=0, k \leqq n$ say, then $(7)$ has to be replaced by

$$
\begin{aligned}
\Pi_{1 \rho} & =\cdots=\Pi_{k \rho}=0, \quad(\rho=1, \cdots, n) . \\
\Pi_{k+1, \rho} / \Pi_{k+1} & =\cdots=\Pi_{n \rho} / \Pi_{n}
\end{aligned}
$$

The equations (7) viz (8) form a necessary condition for the analyticity of $f(x)$ at $x=x_{0}$. We are therefore entitled to consider them as a generalization of the Cauchy-Riemann differential equations.

In the case $n=2$ and complex multiplication, the equations (7) yield the Cauchy-Riemann equations. In the case $n=4, E_{n}$ being a real space and the product being defined according to the multiplication of quaternions, these equations read 


$$
\begin{aligned}
& \phi_{11}=\phi_{22}=\phi_{33}=\phi_{44}, \\
& \phi_{21}=-\phi_{12}=\phi_{43}=-\phi_{34}, \\
& \phi_{31}=-\phi_{42}=-\phi_{13}=\phi_{24}, \\
& \phi_{41}=\phi_{32}=-\phi_{23}=-\phi_{14}
\end{aligned}
$$

where we have abbreviated $\partial \phi_{\nu} / \partial \xi_{\mu}$ by $\phi_{\nu \mu}$. In the case $n=3, E_{n}$ being real and the product being the ordinary vector product, the function $f\left(\xi_{1}, \xi_{2}, \xi_{3}\right)=\left\{\xi_{2}-\xi_{3}, \xi_{3}-\xi_{1}, \xi_{1}-\xi_{2}\right\}$ turns out to be everywhere analytic.

University of Melbourne, Melbourne, Australia 